\title{
Common-Acoustic-Poles and Zeros Modeling Using Jointly Balanced Model Truncation
}

\author{
C. J. Liu and Shih-Fu Hsieh \\ Department of Electrical Engineering \\ National Chiao Tung University \\ Hsinchu, Taiwan 300 \\ Email: cjliu.cm89g@cc.nctu.edu.tw, sfhsieh@mail.nctu.edu.tw \\ Tel: +886-3-5731974, Fax: +886-3-5710116
}

\begin{abstract}
We propose a new technique for commonacoustic-poles and zeros (CAPZ) modeling. In 3-D sound processing, head-related transfer functions (HRTF's) record channels' characteristics from different locations to both ears. Compared to conventional pole/zero models, it is more efficient to model HRTF's by using CAPZ modeling. Based on balanced model truncation (BMT), jointly BMT is proposed to effectively model a group of HRTF's using common-poles IIR filters. The simulation result shows the proposed method is superior to the previous Prony and Shanks methods.
\end{abstract}

Keywords- Nonlinear identification, IIR filters

\section{INTRODUCTION}

Head-related transfer functions (HRTF's) contain sound transmission behavior from different locations to both ears. Such an enormous data set makes real-time implementation for 3 dimensional (3D) sound processing difficult, therefore, approximation of FIR by IIR digital filters is a more efficient way to describe HRTF's. To further save processing time and the memory size, a group of HRTF's can share a set of poles but use their individual zeros, which is called common-acoustic-poles and zeros (CAPZ) modeling. For CAPZ modeling, Prony, Shanks and the iterative prefiltering approaches have been proposed in previous works[3][4]. Based on balanced model truncation (BMT)[1], we propose a new method, jointly balanced model truncation (jointly BMT). BMT is promising in low-order modeling of HRTF's, but it can only determine IIR filter's parameters for individual HRTF's. We extend BMT to jointly BMT so that a group of HRTF's can be simultaneously modeled as IIR filters whose poles are identical.

\section{BALANCED MODEL TRUNCATION}

The details for BMT can be found in [2]. Only a brief outline is given here. We start with an FIR filter $F(z)$ with order $n$ written as:

$$
F(z)=c_{0}+c_{1} z^{-1}+\cdots+c_{n} z^{-n} .
$$

In the state-space model, $F(z)$ can be expressed as a set of difference equations:

$$
\begin{aligned}
x(k+1) & =A x(k)+B u(k) \\
y(k) & =C x(k)+D u(k)
\end{aligned}
$$

where

$$
\begin{gathered}
A=\left[\begin{array}{ccccc}
0 & 0 & \cdots & 0 & 0 \\
1 & 0 & \cdots & 0 & 0 \\
\vdots & \vdots & \ddots & : \vdots & \\
0 & 0 & \cdots & 1 & 0
\end{array}\right] \\
B=\left[\begin{array}{c}
1 \\
0 \\
\vdots \\
0
\end{array}\right] \\
C=\left[c_{1}, c_{2}, \ldots, c_{n}\right],
\end{gathered}
$$

and

$$
D=c_{0}
$$

The transfer function $F(z)$ is related to the statespace model by the formulation

$$
F(z)=C(z I-A)^{-1} B+D \text {. }
$$

$F(z)$ can be approximated by the $k$ th order reduced balanced system $\left(A^{(k)} B^{(k)} C^{(k)} D\right)$ using BMT. A low-order approximated IIR filter $F^{(k)}(z)$ is related to $\left(A^{(k)} B^{(k)} C^{(k)} D\right)$ by 


$$
F^{(k)}(z)=C^{(k)}\left(z I-A^{(k)}\right)^{-1} B^{(k)}+D
$$

where

$$
A^{(k)}=V_{k}^{T} A V_{k}, B^{(k)}=V_{k}^{T} B, C^{(k)}=C V_{k}, D=c_{0} .
$$

To determine $V_{k}$, we can define a Hankel matrix as follows:

$$
H=\left[\begin{array}{cccc}
c_{1} & c_{2} & \ldots & c_{n} \\
c_{2} & c_{3} & \ldots & 0 \\
\vdots & \vdots & \ddots & \vdots \\
c_{n} & 0 & \cdots & 0
\end{array}\right]
$$

Because $H$ is a symmetric matrix, it can be factorized as:

$$
H=V \Lambda V^{T} .
$$

$V_{k}$ is an $n \times k$ matrix obtained from the following partition:

$$
V=\left[V_{k} V_{n-k}\right] .
$$

\section{JOINTLY BALANCED MODEL TRUNCATION}

BMT can only be used to decide the approximated IIR filter for individual HRTF's. In order to design IIR filters for a set of HRTFs which are close spatially, it is not efficient to design different IIR for different HRTF. Based on BMT, jointly BMT is proposed to approximate a group of HRTF's by using common-poles IIR filters.

From (1), we know that the poles of an approximated IIR filter are determined by $A^{(k)}$. From (2), we know that $A^{(k)}$ is determined by $V_{k}$. Therefore, we know that a group of HRTF's can be approximated by one set of common poles and individual sets of zeros if those Hankel matrices corresponding to different HRTF's share a set of truncated eigenvectors $V_{k}$. In the following, we try to find $V$ which can be shared by a group of Hankel matrices because $V_{k}$ is given by (5). In finding eigenvectors of $H$, we try to find $\mathbf{x}_{1}$ that satisfies:

$$
\max _{\left\|\mathbf{x}_{\mathbf{1}}\right\|=1}\left\|H \cdot \mathbf{x}_{\mathbf{1}}\right\|^{2}
$$

where $\mathbf{x}_{\mathbf{1}}$ is the dominant eigenvector corresponding to the largest eigenvalue of $H$. In turn, we can find $\mathbf{x}_{\mathbf{2}}, \ldots, \mathbf{x}_{\mathbf{n}}$ which are orthogonal eigenvectors of $H$.
Now that we are faced with a group of Hankel matrices, $H_{1}, H_{2}, \ldots, H_{N}$, the above eigenvalue problem that seeks the dominant eigenpair of one single Hankel matrix needs to be reformulated. Here we need to find a virtual eigenvector $\mathrm{x}_{1}$ which can satisfy the following maximum criteria simultaneously:

$$
\begin{gathered}
\max _{\left\|\mathbf{x}_{\mathbf{1}}\right\|=1}\left\|H_{1} \cdot \mathbf{x}_{\mathbf{1}}\right\|^{2}, \\
\max _{\left\|\mathbf{x}_{\mathbf{1}}\right\|=1}\left\|H_{2} \cdot \mathbf{x}_{\mathbf{1}}\right\|^{2}, \\
\max _{\left\|\mathbf{x}_{\mathbf{1}}\right\|=1}\left\|H_{N} \cdot \mathbf{x}_{\mathbf{1}}\right\|^{2} .
\end{gathered}
$$

This attempt is not likely to work out successfully unless $H_{1}, H_{2}, \ldots, H_{N}$ have a common set of eigenvectors. As a compromise, we can integrate the above multiple eigenvalue criteria into one single criterion. A jointly BMT model is thus formulated by solving $\mathbf{x}_{\mathbf{1}}$ from the following linearly combined eigenvalue problem:

$$
\begin{gathered}
\max _{\left\|\mathbf{x}_{\mathbf{1}}\right\|=1}\left(\left\|H_{1} \cdot \mathbf{x}_{\mathbf{1}}\right\|^{2}+\left\|H_{2} \cdot \mathbf{x}_{\mathbf{1}}\right\|^{2}+\ldots+\left\|H_{N} \cdot \mathbf{x}_{\mathbf{1}}\right\|^{2}\right) \\
=\max _{\left\|\mathbf{x}_{\mathbf{1}}\right\|=1}\left\|\left[\begin{array}{c}
H_{1} \\
H_{2} \\
\vdots \\
H_{N}
\end{array}\right] \cdot \mathbf{x}_{\mathbf{1}}\right\|^{2}
\end{gathered}
$$

It means that $\mathbf{x}_{1}$ is the dominant eigenvector corresponding to the largest eigenvalue of $\mathcal{H}^{T} \mathcal{H}$, which is the correlation matrix of the cascaded joint Hankel matrices $\mathcal{H}$, denoted as

$$
\mathcal{H}=\left[\begin{array}{c}
H_{1} \\
H_{2} \\
\vdots \\
H_{N}
\end{array}\right]
$$

In Common-Acoustic-Pole-Zero(CAPZ) modeling, there are a group of $N$ HRTF's denoted as $F_{1}(z), F_{2}(z), \ldots, F_{N}(z)$. Each HRTF is measured from different locations to the ear. For each HRTF, we can define a Hankel matrix $H_{i}$ in (3). In order to find a set of eigenvectors shared by all Hankel matrices, we can build a joint Hankel matrix $\mathcal{H}$ by cascading $H_{1}, H_{2}, \ldots, H_{N}$ as given in (6). We 
assume $\mathcal{H}$ is an $m \times n$ matrix $(m>n)$ whose SVD decomposition can be written as

$$
\mathcal{H}=U \Sigma V^{T}
$$

$V$ contains the eigenvectors of $\mathcal{H}^{T} \mathcal{H}$. Finally, each $F_{i}(z)$ can be approximated by the $k$ th order reduced system $\left(A^{(k)} B^{(k)} C_{i}^{(k)} D_{i}\right)$ where

$$
\begin{gathered}
A^{(k)}=V_{k}^{T} A V_{k}, \\
B^{(k)}=V_{k}^{T} B, \\
C_{i}^{(k)}=C_{i} V_{k}, D_{i}=c_{i 0}
\end{gathered}
$$

$i=1,2, \ldots, N$ and $V_{k}$ is given by (5). The choice of order $k$ depends on the distribution of the singular values along the diagonal elements of $\Sigma$ 's. The substantially smaller part of $\Sigma$ 's diagonal elements can be viewed as redundancy which has negligible effect on the system response.

Now we summarize the complete jointly BMT design procedure as follows.

1) given a group of FIR filters $F_{1}(z), F_{2}(z), \ldots, F_{N}(z)$

2) build Hankel matrices $H_{1}, H_{2}, \ldots, H_{N}$ in (3)

3) form a joint Hankel matrix $\mathcal{H}$ in (6)

4) singular value decomposition of $\mathcal{H}$ in (7)

5) display the diagonal elements of $\Sigma$ and decide the order $k$ of reduced system

6) calculate the $\left(A^{(k)} B^{(k)} C_{i}^{(k)} D_{i}\right)$ in (8)

7) convert the $k$ th order reduced system $\left(A^{(k)} B^{(k)} C_{i}^{(k)} D_{i}\right)$ into the transfer function using

$$
F_{i}^{(k)}(z)=C_{i}^{(k)}\left(z I-A^{(k)}\right)^{-1} B^{(k)}+D_{i}
$$

where $F_{i}^{(k)}(z)$ is the approximated IIR filter of $F_{i}(z), i=1,2, \ldots, N$

\section{SIMULATION RESULTS}

The HRTF's we use here are measured by MIT media lab[5]. We choose 14 HRTF's for the left ear as a group with the azimuth $=0$ degree and elevations ranging from -40 degree to 90 degree by 10-degree increase. Before simulation, the HRTF's measured by MIT are prefiltered. Initial time delays are removed and the tails of HRTF's with longer data length are discarded so that all HRTF's are of

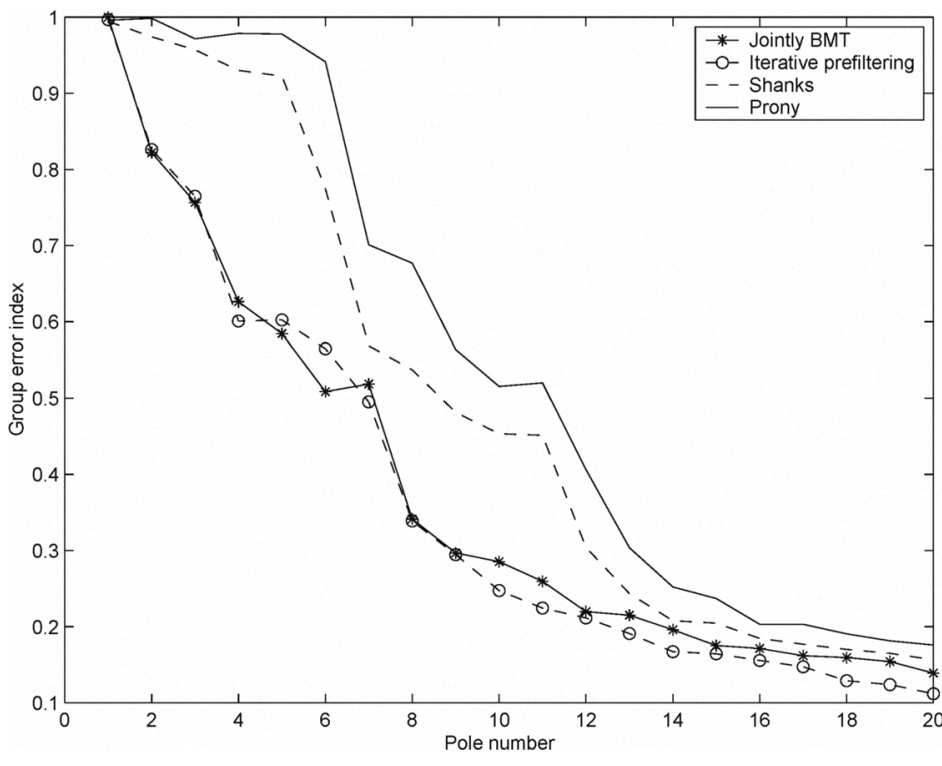

Fig. 1. Comparison of group error indices for four CAPZ models (pole number=zero number)

the same data length. For performance comparison, we define a group error index as:

$\sqrt{\frac{\left\|F_{1}-F_{1}^{(k)}\right\|^{2}+\left\|F_{2}-F_{2}^{(k)}\right\|^{2}+\ldots+\left\|F_{N}-F_{N}^{(k)}\right\|^{2}}{\left\|F_{1}\right\|^{2}+\left\|F_{2}\right\|^{2}+\ldots+\left\|F_{N}\right\|^{2}}}$

where $F_{i}$ is the actual impulse response and $F_{i}^{(k)}$ is the approximated HRTF via different IIR models. In our simulation, we compare group error index performances of 4 different CAPZ models, including Prony, Shanks, the iterative prefiltering, and our proposed jointly BMT methods. For each method , we plot the curve by changing the pole numbers (which is also the zero number). The result in Figure 1 shows that the proposed jointly BMT model is superior to both Prony and Shanks methods, and it is comparable with the iterative prefiltering method.

\section{CONCLUSION}

In real-time applications, processing time is crucial. The proposed method can efficiently model a set of HRTF's. The simulation result shows that the proposed model outperforms the conventional Prony and Shanks methods.

\section{ACKNOWLEDGMENTS}

The authors would like to thank the NSC and MediaTek research center at National Chiao Tung University for their support of this work. 


\section{REFERENCES}

[1] Jonathan Mackenzie, Jyri Huopaniemi, Vesa Valimaki and Izzet Kale, "Low-Order Modeling of Head-Related Transfer Functions Using Balanced Model Truncation," IEEE Signal Processing Letters, vol. 4, no. 2, pp. 39-41, February 1997.

[2] Bartlomiej Beliczynski, Izzet Kale and Gerald D. Cain, "Approximation of FIR by IIR Digital Filters: An Algorithm Based on Balanced Model Reduction," IEEE Transactions on Signal Processing, vol. 40, no. 3, pp. 532-542, March 1992.

[3] Y. Haneda, S. Makino, Y. Kaneda and N. Kitawaka, "CommonAcoustical-Pole and Zero Modeling of Head-Related Transfer Functions," IEEE Trans. on Speech and Audio Processing, Vol. 7, No. 2, p 188-195, March 1999.

[4] M. C. Chen and S. F. Hsieh, "Common Acoustical-Poles/Zeros Modeling for 3-D Sound Processing,"International Conference on Acoustic, Speech and Signal Processing, Istanbul, 2000.

[5] Bill Gardner and Keith Martin,"HRTF Measurements of a KEMAR Dummy-Head Mrcrophone," MIT Media Lab Perceptual Computing-Technical Report, May 1994. 